\title{
LA REPRESENTACIÓN GRÁFICA GEORREFERENCIADA ALTERNATIVA EN LA COORDINACIÓN CATASTRO-REGISTRO EN ESPAÑA. ESTUDIO DE LA IMPLANTACIÓN DE LA NORMA ISO-19152 (LADM) A NIVEL INTERNACIONAL
}

\author{
THE GEORREFERENCED ALTERNATIVE GRAPHIC REPRESENTATION IN THE CADASTRE-LAND REGISTRY \\ COORDINATION IN SPAIN. IMPLEMENTATION STUDY OF THE ISO-19152 (LADM) AT THE INTERNATIONAL \\ LEVEL
}

\author{
Joan Manuel Velilla-Torres*, Gaspar Mora-Navarro, Carmen Femenia-Ribera, José Carlos \\ Martínez-Llario
}

Departamento de Ingeniería Cartográfica, Geodesia y Fotogrametría, Universitat Politècnica de València, Camino de Vera, s/n, Edificio 7i, 46022 Valencia. joavetor@doctor.upv.es; joamona@cgf.upv.es; cfemenia@cgf.upv.es; jomarlla@cgf.upv.es

\begin{abstract}
:
In Spain, the new Law 13/2015, dated June 24, modifying the Mortgage Law and the Cadastre Law in the search for its coordination, introduce the obligation to obtain georeferenced mapping and update the Cadastre in many cases. It is necessary to provide the graphic representation of the farms in case of: segregation, division, grouping and aggregation, and in the case of the operations of parceling, reparcelling, land consolidation, segregation, division, grouping or aggregation, forced expropriation or demarcation which determine a reordering of the land. It also requires the geographical delimitation of the area occupied by buildings declared within a farm. In all of these cases, if there is no coincidence of the farm in the field with the descriptive cadastral certification and the graphic of the farm, an alternative georeferenced graphical representation, duly accredited, is necessary, and where the adventurers are quoted to express or not their Conformity. This thesis aims to investigate an international level with the possibility of collaborating in the current developments, especially applying the ISO 19152 (On the Land Administration Domain Model) to the Spanish case and national level, applying the new Reforms of the Mortgage Law and the Cadastre Law. Performing georeferenced graphical maps, in the cases in which the law forces it. To develop GIS software for the automation of information creation, as well as, the new formats creation used to Spanish Cadastre updating. The software will be tested in the real cases that will be performed.
\end{abstract}

Key words: Reform Mortgage Law, LADM, GeoDelProp, ATNL

\section{Resumen:}

En España, la nueva Ley 13/2015, de 24 de junio, que modifica la Ley Hipotecaria y la Ley del Catastro en la búsqueda de su coordinación introduce la obligatoriedad de obtener cartografía georeferenciada y actualizar el Catastro en muchos casos. Es necesario aportar la representación gráfica de las fincas en caso de: segregación, división, agrupación y agregación, y en caso de que se realicen operaciones de parcelación, reparcelación, concentración parcelaria, segregación, división, agrupación o agregación, expropiación forzosa o deslinde que determinen una reordenación de los terrenos. También exige la delimitación geográfica de la superficie ocupada por las construcciones que se declaren dentro de una finca. En todos estos supuestos, si no hay coincidencia de la finca en el terreno con la certificación catastral descriptiva y gráfica de la finca, es necesaria una representación gráfica georreferenciada alternativa, debidamente acreditada, y donde se cita a los colindantes para que expresen o no su conformidad. En esta tesis se pretende investigar a nivel internacional con la posibilidad de colaborar en los desarrollos actuales, sobre todo aplicando la norma ISO 19152 (Sobre Land Administration Domain Model) al caso español y a nivel nacional, aplicando las nuevas reformas de la Ley Hipotecaria y la Ley del Catastro. Realizando representaciones gráficas georreferenciadas alternativas en casos en los que la ley obliga. Desarrollar aplicaciones SIG para la automatización y generación de la información y de los nuevos formatos necesarios para la actualización del Catastro español, aplicándolo a los casos prácticos que se realicen.

Palabras clave: Reforma Ley Hipotecaria, LADM, GeoDeIProp, ATNL

\section{Reforma de la Ley Hipotecaria $13 / 2015$}

La Ley 13/2015, de 24 de junio, de Reforma de la Ley Hipotecaria aprobada por Decreto de 8 de febrero de 1946 y del texto refundido de la Ley de Catastro
Inmobiliario, aprobado por Real Decreto Legislativo $1 / 2004$, de 5 de marzo, ha sido publicada en el BOE el 25 de junio de 2015.

"Corresponding Author: Joan Manuel Velilla-Torres, joavetor@doctor.upv.es 
Esta ley tiene como fin resolver varios problemas que presentaba la regulación existente, y como bases de esta reforma se puede anunciar que:

Anteriormente, la delimitación de los deslindes de las fincas registradas era potestativa, es decir, no era obligatoria, permitiendo bases gráficas no georreferenciadas. Con la reforma, la descripción gráfica de las fincas registrales será obligatoria en los supuestos de modificación de la configuración territorial de las fincas registrales. En el resto de casos será voluntaria. Esta representación gráfica deberá tener georreferenciada su delimitación en un sistema oficial, ya que permitirá distinguirla de las colindantes, ubicarla y delimitarla sobre el territorio.

La cartografía catastral se constituye como base gráfica del Registro de la Propiedad para la descripción de las fincas, según el artículo 10.

Se regulan todos los procedimientos de inscripción en el Registro de la Propiedad para garantizar la coordinación con el Catastro inmobiliario (Berné 2004). Los procedimientos de coordinación se regulan de manera muy garantista para evitar perjuicios a terceros y colindantes.

Se debe de aportar, junto a la justificación catastral descriptiva y gráfica, la descripción de la finca que hacen los Registradores indicando si está coordinada o no gráficamente con el Catastro. Por lo que, la creación de la figura de la finca coordinada gráficamente con el Catastro, tendrá efectos jurídicos reforzados (presunción de certeza) tras la calificación del registrador. A continuación se ha de trasladar al catastro el código registral único de la finca cuando se alcance esta coordinación.

Se regula de manera detallada la interoperabilidad entre el Catastro y el Registro de la Propiedad. El proceso de coordinación será paulatino a medida que se inscriben en el Registro los nuevos actos o negocios donde se reflejen alteraciones de las fincas.

La representación gráfica alternativa se realizará cuando la cartografía del catastro no se corresponda con la realidad o cuando la finca surja al tráfico jurídico, y que, por lo tanto, tampoco exista en el catastro.

\subsection{Representación Gráfica Alternativa (RGA)}

- Permite a los interesados incorporar las alteraciones que se produzcan en los inmuebles, o rectificar la cartografía catastral por los procedimientos establecidos.

- Debe ser de aprobada expresamente por el propietario o interesados.

- Tiene que ser representada sobre la cartografía catastral.

- Se debe representar las coordenadas georreferenciadas de los vértices en todos sus elementos.

- Tiene que hacerse en un fichero informático con soporte GML, y en el formato de parcela catastral INSPIRE.
- Indicar la fecha de su realización y su metodología.

- Que es responsabilidad, del técnico que haya realizado la representación geográfica, que sean ciertas las condiciones técnicas con las que describe su trabajo.

- La resolución se podrá obtener mediante levantamiento topográfico del terreno, pero no será obligatorio.

- $\quad$ El sistema de referencia es el ETRS89 en la península, y REGCAN en las Islas Canarias, en la proyección universal transversal de Mercator UTM.

Para una validación gráfica de la RGA se utiliza el servicio automatizado en la Sede Electrónica del Catastro (SEC). Si esta validación es positiva, se obtiene un informe de validación grafica (IVG). EI IVG es un informe técnico que valida gráficamente la RGA elaborada por los interesados, pero no valida la legalidad de la operación que se propone. EI IVG es potestativo y no vinculante.

El fichero GML (Geography Markup Language) (Zhang et al. 2011) debe de estar firmado electrónicamente por el técnico competente, y tiene que contener el Identificador único del objeto, sistema de referencia, listado de vértices que definen la poligonal cerrada terminando y empezando las listas de los vértices siempre en el mismo vértice. Si la parcela catastral tiene huecos se añaden estructuras con su geometría, referencia catastral, país y organismo, etiqueta, referencia catastral nacional y las coordenadas del centroide.

Nueva regularización de la inscripción de las edificaciones en el Registro de la Propiedad.

Antes de la ley 13/2015, cuando había una edificación el técnico certificaba de la antigüedad de la edificación que incorporaba en el documento público y se llevaba al registro.

Ahora, según el Art 102, la porción del suelo ocupada por cualquier edificación, instalación o plantación tiene que estar identificada mediante sus coordenadas de representación geográfica, y en el caso de que se trate de edificaciones en régimen de propiedad horizonta, se hará constar en el folio real de cada elemento, independiente su respectiva representación gráfica, tomada del proyecto incorporado al libro el edificio.

\section{Delimitación topográfica de un finca registral aplicando la Reforma de la Ley Hipotecaria}

Para poner en práctica dicha Reforma de la Ley Hipotecaria, se realizó una delimitación topográfica de una finca registral situada en el municipio de Montserrat, ya que dicha finca, por una equivocación informada en su día en el Catastro, en la actualidad aparecía grafiada con un desplazamiento a una parcela distinta a la que realmente corresponde. En concreto aparece en la parcela 205 cuando en realidad su ubicación es en la parcela 376. En la Figura 1 se demuestra, con un dibujo aproximado en el que el propietario identificó dicho desplazamiento: 


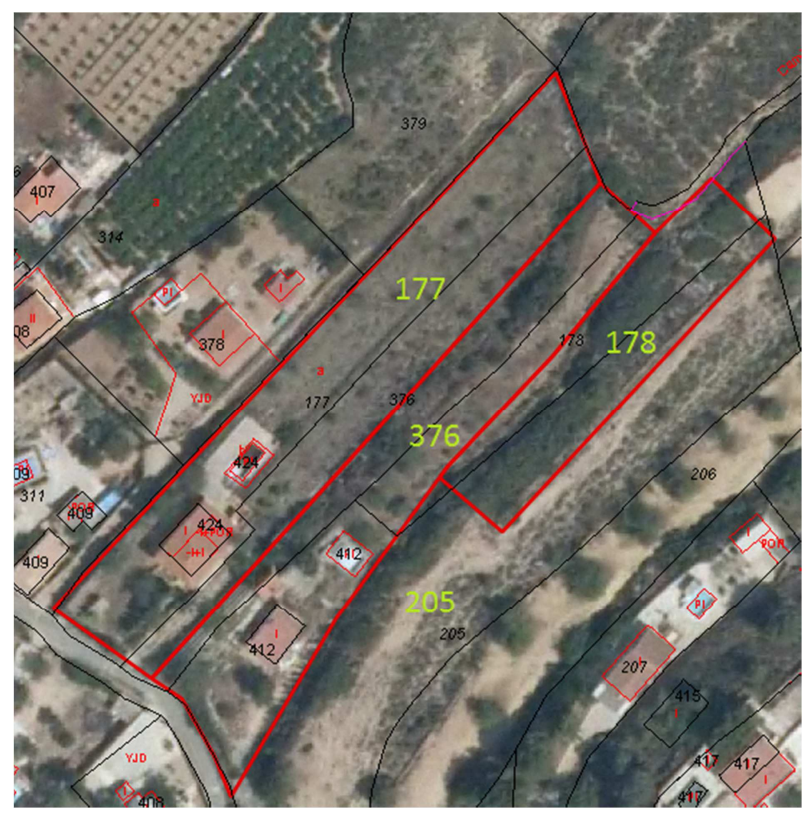

Figura 1: Parcelas a delimitar en el Proyecto.

Las líneas y textos en negrita corresponden a las delimitaciones y anotaciones de la cartografía catastral. Las líneas en rojo corresponden a una aproximación de la delimitación topográfica correcta de las fincas catastrales, y las anotaciones en amarillo a la ubicación correcta de las parcelas catastrales. Se observa que las delimitaciones de las parcelas 376 y 178, según el catastro, no corresponde con las delimitaciones de la realidad dibujadas en rojo, quedando parte de la finca de la parcela 376 dentro de la parcela 205. La parcela 178 pertenece al Ayuntamiento de Montserrat.

Para la delimitación topográfica de la finca, se realiza una medición topográfica con GPS de todos sus linderos. Durante la medición acudió un representante del propietario, estando de acuerdo en dicho trabajo en la toma de puntos de los lindes junto a sus respectivas fotografías.

\subsection{Equipo utilizado y precisiones}

El levantamiento topográfico de este trabajo, se ha realizado con tecnología RTK con solución de red VRS. Esta técnica alcanza precisiones de hasta $2 \mathrm{~cm}$, de desviación típica, en el sistema de referencia ETRS89. El equipo utilizado ha sido el GPS Leica System 1200. Por razones de rendimiento, se configura el equipo para asegurar una precisión de 5 centímetros, en el sistema de coordenadas EPSG 25830.

\subsection{Cartografía existente en el trabajo}

Cartografía catastral, obtenida de la SEC, en la fecha del levantamiento. Se utiliza para comprobar la coincidencia del levantamiento con la cartografía catastral.

Cartografía del PNOA (Plan Nacional de Ortofotografía Aérea), a partir de servicios WMS se obtiene una imagen recortada de dicha cartografía en la zona de trabajo, que georreferenciándola e incorporándola al proyecto se utiliza como fondo del levantamiento, para que el propietario reconozca los elementos existentes en el terreno, junto a las líneas de los linderos dibujadas.

Planos de la Cartografía histórica catastral, producida por el entonces Instituto Geográfico Catastral, el Mapa Topográfico Parcelario (MTP).

Plano topográfico antiguo, producido por Jose Soler Trilla colegiado del Colegio Oficial de Ingenieros Técnicos en Topografía, realizado el 1977, a escala $1 / 500$.

\subsection{Importación a GeoDelProp}

Una vez realizado el levantamiento topográfico, se publican sus datos espaciales, tanto geometrías como metadatos, con software GeoDelProp, basado en el software TopoDelProp (Mora-Navarro 2013). Con esto, se pretende ayudar a Notarios, Registradores y ciudadanos a identificar sus lindes sobre el terreno, de forma que se añade seguridad al proceso de delimitación. Este software permite generar y descargar los ficheros GML INSPIRE con las geometrías de los elementos espaciales para una validación en el Catastro (Fig. 2).

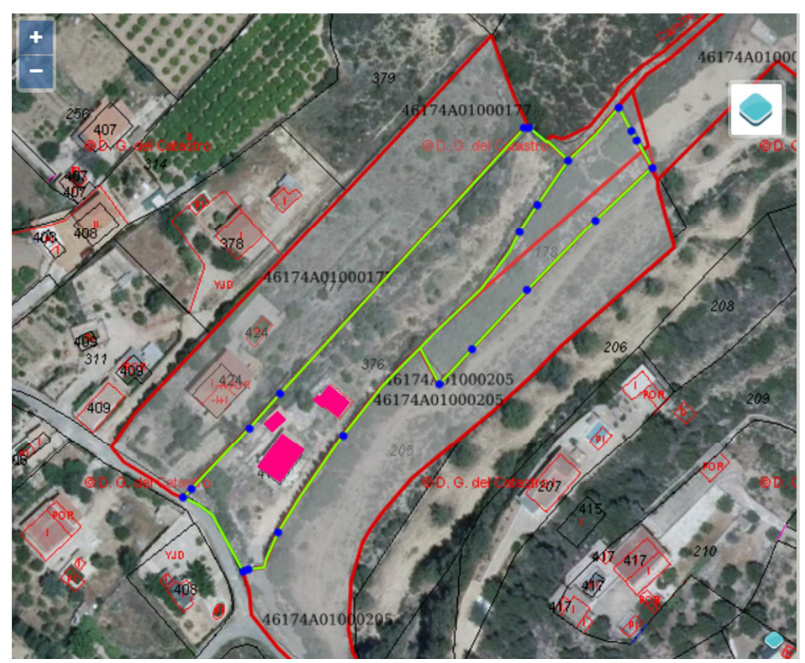

Figura 2: Publicación en el geoportal GeoDelProp (http://upvusig.car.upv.es/geodelprop/).

\subsection{Actualización cartografía en el Catastro}

La actualización de la cartografía en el Catastro se pretendía realizar a través de una Notaría siguiendo los procedimientos que marca la nueva Ley, pero siendo tan reciente la norma (pocos meses) no están preparados todos los cauces necesarios para realizar correctamente el procedimiento a través de la Notaría. Por ello, en este caso, se optó por realizar la actualización de la cartografía catastral aportando el GML directamente a Catastro ya que corría prisa y de este modo se comprobaba la actualización.

Destacar que, la aportación de GML por los técnicos utilizando los IVG del Catastro está contemplado en la nueva Ley siempre que se realicen a través de Notarías y Registros; pero no contempla el caso de aportarlos directamente a Catastro. En caso de realizar la actualización y coordinación gráfica entre Catastro y Registro la coincidencia gráfica con la RGA del técnico debe ser exacta. 
Tras entregar la información directamente a Catastro finalmente se obtienen los resultados de la actualización de la cartografía catastral quedando, como se observa en las Figuras 3 y 4.

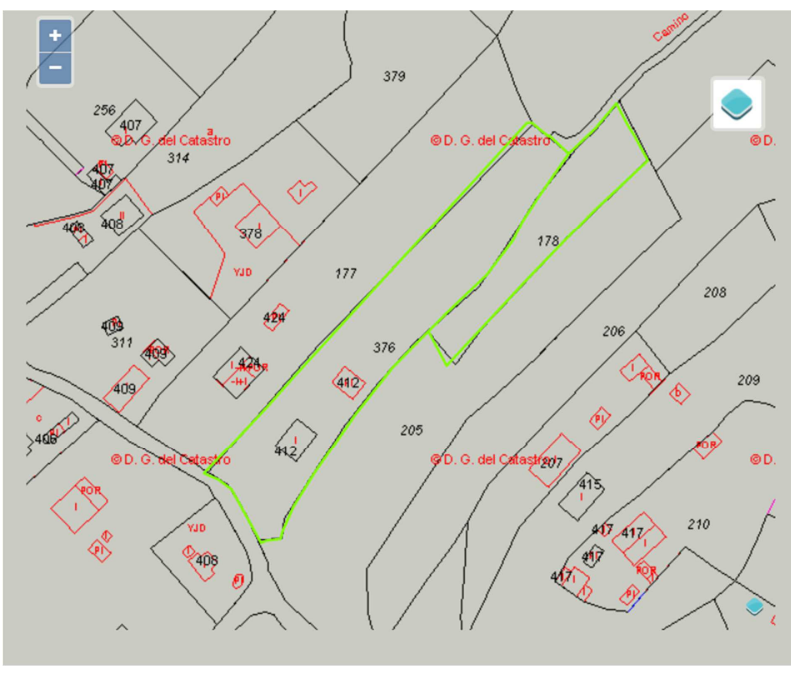

Figura 3: Resultado tras la actualización del Catastro.

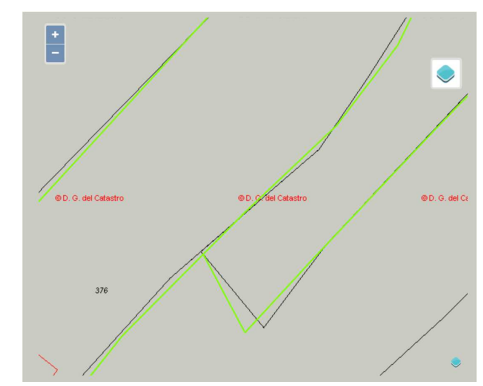

Figura 4: Zoom al resultado tras la actualización del Catastro.

Las líneas en verde corresponden a los lindes del levantamiento topográfico realizado, y las líneas en negro y rojo corresponden a la cartografía catastral junto con sus anotaciones. La cartografía catastral no coincide exactamente con la geometría del levantamiento topográfico, ello es debido a que no se ha tramitado la información gráfica a través de la Notaría y/o Registro; en este caso la coincidencia sería total. Al tramitarlo directamente a través de Catastro las pequeñas variaciones en la cartografia entran en tolerancia técnica 0 no es necesaria subsanarlas. A pesar de que existen diferencias que no encajan perfectamente, el propietario de la parcela catastral está conforme con el resultado, considerando que es suficiente para la finalidad planteada.

Con este trabajo práctico se puede concluir que se ha conseguido investigar y estudiar la Reforma de la Ley Hipotecaria aplicándola a un caso en concreto obteniendo unos resultados satisfactorios. Se ha realizado un levantamiento topográfico de una finca con una precisión de 5 centímetros en las coordenadas tomadas. La publicación en el software GeoDelProp facilita a Notarios, Registradores, propietarios y vecinos identificar los lindes y crear el archivo GML para validación en el Catastro. El desarrollo de este proyecto se ha concluido con un informe para el Registrador de la Propiedad junto con sus planos. Un ejemplo estos planos es el representado en la Figura 5, donde se indica en color azul la delimitación resultante de las fincas número 376 y 178 tomadas en el levantamiento junto con las coordenadas de sus puntos.

En la Figura 5 se muestra la cartografía catastral de las fincas, en color rojo, y el levantamiento realizado, donde realmente deberían estar situadas, en color azul.

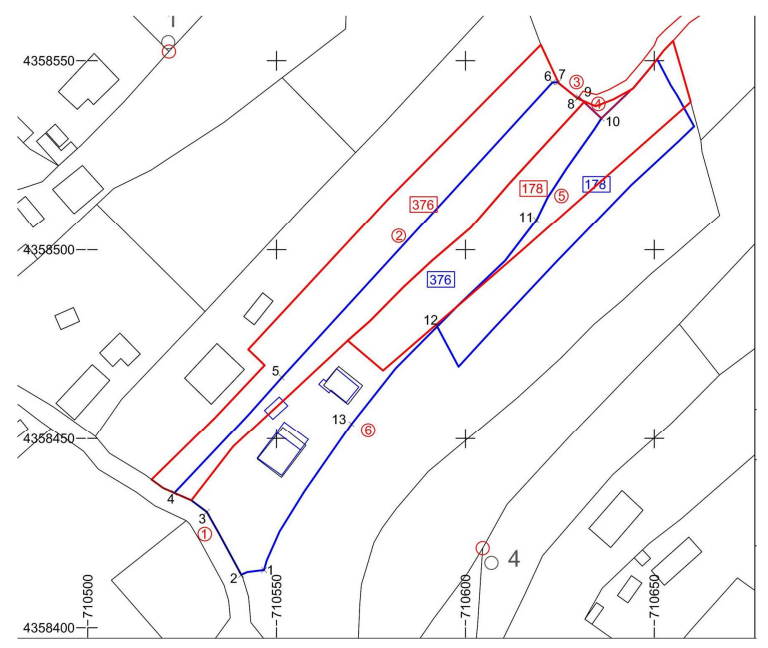

Figura 5: Plano de coordenadas del levantamiento topográfico.

Las coordenadas UTM han sido tomadas en el huso 30 , en el sistema de referencia ETRS89 con un error máximo en cada punto de $0.1 \mathrm{~m}$ (Fig. 6). Los elementos divisores de las fincas se han medido según las señales existentes en el terreno: muros, vallas, caminos etc...

\begin{tabular}{|c|c|c|c|}
\hline Pto & $X$ & $Y$ & Precisión \\
\hline 1 & 710546,68 & 4358415,23 & Catastro \\
\hline 2 & 710540,65 & 4358413,95 & Catastro \\
\hline 3 & 710531,52 & 4358430,57 & Catastro \\
\hline 4 & 710522,93 & 4358435,58 & Catastro \\
\hline 5 & 710551,37 & 4358466,26 & 0,1 \\
\hline 6 & 710623,05 & 4358544,28 & 0,1 \\
\hline 7 & 710624,73 & 4358544,08 & Catastro \\
\hline 8 & 710629,75 & 4358540,08 & Catastro \\
\hline 9 & 710629,99 & 4358540,41 & 0,1 \\
\hline 10 & 710636,00 & 4358534,81 & 0,1 \\
\hline 11 & 710618,80 & 4358507,90 & 0,1 \\
\hline 12 & 710592,51 & 4358479,69 & 0,1 \\
\hline 13 & 710569,83 & 4358453,92 & 0,1 \\
\hline
\end{tabular}

\begin{tabular}{|c|c|}
\hline PARCELA & ÁREA FINAL \\
\hline 178 & 1254,20 \\
\hline 376 & 3303,66 \\
\hline
\end{tabular}

Figura 6: Coordenadas del levantamiento topográfico.

El trámite tradicional a través de Catastro es suficiente en determinados casos, aunque si se pretende una mayor rapidez y precisión en la representación gráfica lo mejor es tramitar los procedimientos adecuados a través de Notarías y Registros aplicando la nueva Ley. En estos momentos (mayo 2017) aún no están totalmente operativos los procedimientos que permitan actualizar 
rápidamente las RGA que procedan de Notaría y Registros, pero en el momento en que los estén se pretende que sean prácticamente automáticos y permitan modificar parcelas catastrales en días, incluso minutos, y con la misma precisión con la que ha medido el técnico. Se espera que en unos pocos meses sea posible.

\section{ATNL - Archivo Topográfico Nacional de Lindes del Colegio Oficial de Ingenieros en Geomática y Topografía}

El objetivo del ATNL es tener un geoportal que publique geometrías y metadatos topográficos de delimitaciones realizadas por ingenieros colegiados, con el principal objetivo de añadir seguridad al tráfico inmobiliario, basado en la facilidad de comprensión, y de acceso a los datos y metadatos, del trabajo de delimitación, que se consigue con esta plataforma (Fig. 7). Los destinatarios de esta información son notarios, registradores y usuarios afectados por la delimitación.

En este geoportal se puede observar y consultar la información gráfica y alfanumérica de los lindes obtenidos en cada delimitación. Esta base de datos está compuesta por los trabajos de deslindes realizados por los colegiados, que han decidido incorporarlos al ATNL.

El proyecto ATNL se ha basado en los proyectos TopoDelProp y GeoDelProp, desarrollados en la Universidad Politécnica de Valencia.

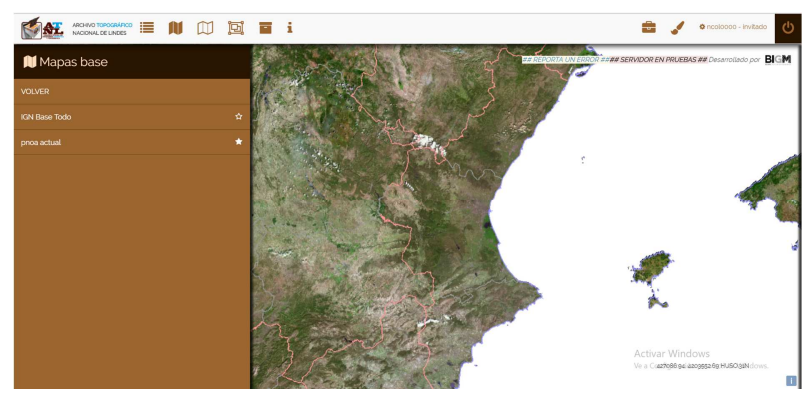

Figura 7: Visor Cartográfico ATNL.

Se pretende próximamente, con la colaboración de notarios y registradores, probar el proyecto ATNL para identificar y delimitar varias fincas registrales colindantes. De este proyecto se espera obtener importantes conclusiones, como el abaratamiento de costes cuando se deslindan grupos de fincas, la utilidad de las imágenes de campo, de la facilidad de acceso a los datos geográficos, de la superposición de las geometrías obtenidas sobre otras cartografías oficiales, para contrastar sus diferencias con el deslinde, etc.

\section{LADM - Land Administration Domain Model - ISO 19152}

La norma ISO 19152 (Land Administration Domain Model) (Alkan and Polat 2016) es un marco internacional de un modelo de datos conceptual y genérico para la administración de tierras en cualquier país (Babalola 2015). Está basado en el marco conceptual de Catastro 2014 de la Federación Internacional de Geómetras (FIG) siguiendo los estándares desarrollados por ISO y coordinado con otras normativas de estandarización especialmente la INSPIRE y también LPIS y STDM.

La LADM desarrolla dos aspectos utilizando el lenguaje de modelo UML (Jiao et al. 2006) en diagramas de clases: el primero de ellos relativo a los derechos, responsabilidad y restricciones (RRR, Right, Responsabilities y Restrictions), que afectan a la superficie terrestre o de agua. El segundo aspecto está referido a los componentes geométricos y espaciales a los que afecta el dominio anterior. Consolida la relación entre el elemento físico (parcela) y derecho, en la cual, la parcela catastral es la descripción del ámbito espacial del derecho de propiedad, lo que lleva a la conclusión de que el Catastro ha de ser la base gráfica del Registro de la Propiedad.

Sus objetivos principales son:

- Evitar la repetición de funcionalidades y así proveer de una base extensible con el fin de desarrollar y mejorar de forma eficiente $y$ efectiva conjuntos de sistemas para administradores del territorio basados en una arquitectura de modelos.

- Permitir a todas las partes que trabajen con el territorio, ya sea un país o en diferentes países, un vocabulario común que está implícito en el modelo.

- Las cuatro clases que se divide la LADM (Velasco 2016) siguen el esquema clásico del catastro, que son:

- LA_Party: Desarrolla las clases relativas a partes (personas u organizaciones) o grupos de partes (grupos de personas $u$ organizaciones).

- LA RRR: Clases para el desarrollo de Derechos, Responsabilidades y Restricciones.

- LA_LAUnit: Este paquete desarrolla la información administrativa relativa a unidades espaciales con los mismos derechos, restricciones o responsabilidades.

- LA_SpatialUnit: Este paquete desarrolla las unidades espaciales, parcelas, subparcelas, edificaciones o redes.

\subsection{Casos donde se aplique la LADM}

\subsubsection{Argentina, proyecto IDERA (Datos} Espaciales de la República Argentina)

Los objetivos del proyecto se basan en:

- $\quad$ El tipo de información que debe disponerse.

- La interoperabilidad entre las IDEs provinciales, locales y organizacionales, ya que cada provincia de Argentina tiene desarrollada su propia IDE.

- El intercambio de información, conocimientos, herramientas de libre acceso.

- Herramientas necesarias para implementar la IDE y desarrollarlas. 
- La implementación gradual de un nodo IDE en cada una de las jurisdicciones, con sus respectivos municipios incluidos o como nodos independientes de acuerdo a la disponibilidad de recursos humanos y tecnológicos.

- El acceso libre y gratuito a la información geoespacial.

Se pretende consensuar entre las distintas organizaciones catastrales un único diccionario de datos, que permita un sistema interoperable de información territorial, a partir de la cual se asegura el intercambio de información generada por cada una de las instituciones. Para ello, la cartografía catastral identificará el objeto territorial legal, es decir, la parcela, a través de su ubicación física y su descripción geométrica individual.

\subsubsection{Colombia, Catastro multipropósito}

El problema en Colombia es originario de un patrón inadecuado de la ocupación y uso de la tierra. Muchos campesinos han identificado su parcela en lugares equivocados, teniendo debilidad en los derechos de esta propiedad, y se han formado atrasos en material social y económico conjuntamente con debilidades institucionales.

El Catastro multipropósito, (Guzman-Jiménez 2017) es la herramienta que permitirá la administración, gestión y gobernanza de las tierras rurales y tener el conocimiento de los predios y de sus tenedores. Permitirá tener en tiempo real toda la información de un bien inmueble y así evitar que existan varios propietarios o un propietario sin documentación acreditativa. Incorpora el análisis de cuatro variables:

- La económica para lograr estimar valores catastrales que reflejen de manera precisa las condiciones físicas y económicas de los bienes inmuebles.

- La identificación y descripción física y jurídica de los bienes inmuebles públicos y privados del país, bajo los estándares definidos y adoptados por la autoridad catastral.

- La jurídica que busca garantizar la anhelada interrelación catastro-registro y contar con una norma integradora y unificadora en estas materias. Esta variable es sin duda, una de las mayores problemáticas que tienen el sector de tierras en Colombia.

- La Institucional para lograr fortalecer la institucionalidad catastral para la implementación del catastro multipropósito y la adecuación de mecanismos para mejorar el acceso e interoperabilidad de la información sobre la tierra y la propiedad.

Para esta implementación del Catastro Multipropósito se diseñaron dos fases, una piloto del nuevo modelo en 11 municipios, la cual estaba prevista para el año 2016, y escalamiento a todo el territorio nacional entre 2017 y 2023. La meta a 2018 es conformar e implementar el catastro multipropósito en el $25 \%$ del país (con énfasis en zonas rurales), y sentar las bases para completar el ejercicio nacional en 2023. El costo de esta política es de $\$ 2,6$ billones y su implementación será liderada por Presidencia de la República, el Departamento Nacional de Planeación (DNP), el Instituto Geográfico Agustín Codazzi (IGAC), la Superintendencia Notariado y Registro (SNR) y la Agencia Nacional de Tierras (ANT).

El Catastro Multipropósito utiliza INTERLIS que es un lenguaje de esquema conceptual para la descripción de modelos especializado en modelos geoespaciales, puesto que incluye varios tipos de geometrías. El formato de transferencia de datos es el ITF o XTF. La principal ventaja es la "libertad de métodos". Es importante el soporte de los conceptos de Catastro 2014, especialmente en cuanto al manejo de la independencia temática con modelos por temas pero dentro de un mismo sistema de referencia; adicional a su flexibilidad para crear modelos de datos en general.

\subsection{Softwares que apliquen el LADM}

\subsubsection{STDM - Social Tenure Domain Model}

STDM (Griffith-Charles 2011) se basa en una plataforma de componentes de código abierto incluyendo PostgreSQL, PostGIS y QGIS. QGIS implementa la biblioteca geoespacial GDAL / OGR, lo que significa que más de 75 formatos vectoriales se pueden importar 0 exportar desde la base de datos STDM.

Esto incluye:

- Bases de datos espaciales PostGIS.

- Formatos de datos vectoriales OGR.

- Bibliotecas de Python geoespaciales.

EI STDM es una "especialización" de la ISO aprobada por la LADM. En este contexto, la especialización significa que hay algunas diferencias, que están sobre todo en la terminología y en el área de aplicación. El desarrollo de LADM tuvo lugar en paralelo con el desarrollo de STDM como concepto y modelo, y que los desarrolladores principales de ambos modelos son los mismos o de apoyo del uno al otro. Cualquier forma de derecho, responsabilidad o restricción en un sistema formal se considera como una relación de tenencia social en STDM.

\subsubsection{SOLA - Solutions for Open Land Administration}

Las estructuras de datos subyacentes utilizadas por el software SOLA se basan en el Modelo de Dominio de Administración de Tierras (LADM).

El interés en el proyecto SOLA sigue aumentando y la FAO ha seguido apoyando al proyecto SOLA, mediante la asistencia de que disponen los países que desean aplicar las Directrices voluntarias sobre la gobernanza responsable de la tenencia (VGGT). En este nuevo contexto, SOLA es visto como un excelente ejemplo de una tecnología habilitadora que mejora la transparencia y la prestación de servicios en las agencias de administración de tierras - elementos clave para mejorar la gobernanza de la tenencia de la tierra. La Unión Europea ha ofrecido su apoyo a través de la VGGT financiando las versiones árabe, francesa y rusa de SOLA, así como el trabajo preliminar para la ampliación de la valoración de la propiedad en masa. Traducciones 
españolas, portuguesas y chinas del software SOLA original (ahora llamado Registro) también están disponibles

EI software SOLA permite un registro original para agencias de administración de tierras que requieren una solución de tenencia segura, robusta y transparente. Esto proporciona un registro integrado con sus respectivas funciones catastrales, gestión de casos y una base de datos compatible con LADM.

\subsubsection{SIGIT - Sistema de Gestión Integral de Tierras}

El Sistema de Gestión Integral de Tierras, SIGIT (Lemmen et al. 2013), es un proyecto piloto que está desarrollado en el marco del Programa URBAL III, Proyecto Gestión Integral de Tierras, con fondos de la Comunidad Europea y asesorado técnicamente por Kadaster de Holanda. El objetivo del sistema es formar una plataforma general para la gestión integral de tierras a nivel local con enlaces con las instituciones a nivel nacional. Actualmente está instalado y en pruebas en los países de Honduras y Guatemala. El SIGIT se caracteriza por:

- Integración de tenencia, uso y planificación de tierras

- Entorno controlado de flujos de trabajo (todo cambio es producto de una transacción, toda transacción es soportada por documentos, toda transacción es trazable hasta sus orígenes, el soporte de historia)

- Integración con otros aplicativos que usan a la unidad territorial como base de operación, haciendo uso de servicios WMS y WFS

- Uso del Land Administration Domain Model para la administración de tierras

- Uso de software fuente abierta

SIGIT funciona de la siguiente manera: los usuarios desde la plataforma web acceden a su municipio con un formulario de solicitud. En el caso de transacciones catastrales, esto incluye documentos notariales y que se pueden incluir como en el caso de una subdivisión. Desde el SIGIT, tras la recepción, la verificación y predicción de los datos de la transacción, se proporcionará un Certificado Catastral en papel. El usuario tomará el certificado, la escritura y (si es necesario) el formulario al NPI (Instituto de Propiedad Nacional). El municipio enviará los datos de transacción digitalmente de SIGIT a SURE (Sistema Unificado de Registros). Entonces NPI puede comparar ambos conjuntos de datos (desde el usuario y desde SURE). Una transacción se procesará en SURE y el municipio será informado digitalmente después de la aprobación por el NPI de esta transacción en SURE.

\section{Conclusiones}

Los estudios planteados de la Reforma de la Ley Hipotecaria y a partir de la plataforma GeoDelProp con el software TopoDelProp, se ha conseguido finalizar un proyecto real de delimitación de una propiedad, obteniendo una validación grafica catastral aceptada y unos resultados satisfactorios para el propietario.

El siguiente objetivo que se pretende alcanzar es utilizar la plataforma ATNL, como se ha mencionado anteriormente, para realizar una delimitación múltiple de fincas y comprobar si la delimitación de grupos de fincas ofrece ventajas. Se plantea una delimitación de entre 10 y 20 fincas colindantes.

Se debe reconocer el LADM como estándar ISO que inicia un desarrollo progresivo de nuevos catastros, integrados con los registros de la propiedad, es decir, a la integración organizacional (registros de la propiedad y oficinas de catastros), que generalmente están separados, contribuyendo también a disminuir las bases de datos redundantes.

Varios países como Colombia y Argentina, ya han personalizado el esquema LADM para sus necesidades: eliminando, editando o añadiendo nuevos elementos para establecer una norma consolidada entre elementos físicos (parcelas) y derecho. Se continua con la tendencia establecida de INSPIRE que sigue en la posición en que la parcela catastral es la descripción del ámbito espacial del derecho de propiedad, como se ha mencionado en el apartado 4 , el Catastro ha de ser la base gráfica del Registro de la Propiedad.

\section{Agradecimientos}

Este trabajo ha sido apoyado por los profesores MoraNavarro, Femenia-Ribera, Martínez-Llario que pertenecen al departamento de Ingeniería Cartográfica, Geodesia y Fotogrametría, Universidad Politécnica de Valencia, España.

\section{References}

ALKAN, M. and POLAT, Z.A., 2016. Design and development of LADM based infraestructura for Turkey. Survey Review, pp. 1-16.

ATNL, 2017. Archivo Topográfico Nacional de Lindes. Available: http://atnl.coigt.com/

BABALOLA, S.O., RAHMAN, A.A., CHOON, L.T. and VAN OOSTEROM, P.J.M., 2015. Possibilities of Land Administration Domain Model (LADM) implementation in Nigeria. Joint International Geoinformation Conference, II2M2, pp. 28-30.

BERNÉ VALERO, J.L, FEMENIA RIBERA, C. and AZNAR BELLVER, J., 2004. Catastro y Valoración Catastral. $1^{2}$ edición. Editorial Universidad Politécnica de Valencia. Ref., pp. 532-574. ISBN: 84-9705-672-8.

GEODELPROP, 2016. Available: http://upvusig.car.upv.es/geodelprop/

GRIFFITH-CHARLES, C., 2011. The Application of the Social Tenure Domain Model (STDM) to Family Land in Trinidad and Tobago. Land Use Policy, 28(3), pp. 514-522. 
GUZMAN-JIMÉNEZ, L.F., 2017. El Catastro Multipropósito: Retos y oportunidades para el desarrollo rural. Available: http://lasillavacia.com

INSPIRE, 2007. Directive 2007/2/EC of the European Parliament and of the Council of 14 March 2007 establishing an Infrastructure for Spatial Information in the European Community (INSPIRE). Available: http://inspire.jrc.ec.europa.eu/

ISO/TC211, 2011. ISO 19152, Draft International Standard (DIS), Geographic information - Land administration domain model (LADM). Lysaker, Norway: ISO, 118 pages.

JIAO, J., ZHANG, L. and HELO, P.T, 2006. Process platform representation based on Unified Modelling Language. International Journey of Production Research, 45 (2007), pp. 323-350.

LEMMEN, C., KOERS, J. and CERRATO-ESPINAL, R., 2013. SIGIT: an Information System for Integral Approach of Land Management: an LADM Implementation in Honduras and Guatemala. 5th Land Administration Domain Model Workshop.

MORA-NAVARRO, G., 2013. Diseño y desarrollo de un modelo de datos dinámico con software libre para la delimitación gráfica de las propiedades en España. Thesis, (PhD). Universidad Politécnica de Valencia.

SIGIT, 2013. Sistema de Gestión Integral de Tierras. Available: http://puertocortes.gob.hn/sigit/home.jsf

VELASCO MARTÍN-VARÉS, A., 2016. La norma ISO TC 211 19152, sobre el Modelo Catastral. Land Administration Domain Model (LADM). Catastro N 87, pp. 7-33.

ZHANG, C., LI, W., DAY, M.J. and PENG Z.R., 2011. GML-Based Interoperable Geographical Databases. Cartography, 32 (2003), pp. 1-16. 